\title{
A nomogram incorporating functional and tubular damage biomarkers to predict the risk of acute kidney injury for septic patients
}

Jianchao Ma ${ }^{1 \dagger}$, Yujun Deng ${ }^{2 \dagger}$, Haiyan Lao ${ }^{3}$, Xin Ouyang ${ }^{4}$, Silin Liang ${ }^{4}$, Yifan Wang ${ }^{4}$, Fen Yao ${ }^{4}$, Yiyu Deng ${ }^{2 *}$ and Chunbo Chen ${ }^{4,5^{*}}$

\begin{abstract}
Background: Combining tubular damage and functional biomarkers may improve prediction precision of acute kidney injury (AKI). Serum cystatin C (sCysC) represents functional damage of kidney, while urinary N-acetyl- $\beta$-Dglucosaminidase (UNAG) is considered as a tubular damage biomarker. So far, there is no nomogram containing this combination to predict AKI in septic cohort. We aimed to compare the performance of AKI prediction models with or without incorporating these two biomarkers and develop an effective nomogram for septic patients in intensive care unit (ICU).

Methods: This was a prospective study conducted in the mixed medical-surgical ICU of a tertiary care hospital. Adults with sepsis were enrolled. The patients were divided into development and validation cohorts in chronological order of ICU admission. A logistic regression model for AKI prediction was first constructed in the development cohort. The contribution of the biomarkers (sCysC, uNAG) to this model for AKI prediction was assessed with the area under the receiver operator characteristic curve (AUC), continuous net reclassification index (cNRI), and incremental discrimination improvement (IDI). Then nomogram was established based on the model with the best performance. This nomogram was validated in the validation cohort in terms of discrimination and calibration. The decision curve analysis (DCA) was performed to evaluate the nomogram's clinical utility.
\end{abstract}

(Continued on next page)

\footnotetext{
*Correspondence: yiyudeng666@163.com; gghccm@163.com

${ }^{2}$ Department of Critical Care Medicine, Guangdong Provincial People's

Hospital, Guangdong Academy of Medical Sciences, 510080 Guangzhou, Guangdong Province, PR China

${ }^{4}$ Department of Intensive Care Unit of Cardiovascular Surgery, Guangdong Cardiovascular Institute, Guangdong Provincial People's Hospital, Guangdong Academy of Medical Sciences, 106 Zhongshan Er Road, 510080 Guangzhou, PR China

Full list of author information is available at the end of the article
}

(c) The Author(s). 2021 Open Access This article is licensed under a Creative Commons Attribution 4.0 International License, which permits use, sharing, adaptation, distribution and reproduction in any medium or format, as long as you give appropriate credit to the original author(s) and the source, provide a link to the Creative Commons licence, and indicate if changes were made. The images or other third party material in this article are included in the article's Creative Commons licence, unless indicated otherwise in a credit line to the material. If material is not included in the article's Creative Commons licence and your intended use is not permitted by statutory regulation or exceeds the permitted use, you will need to obtain permission directly from the copyright holder. To view a copy of this licence, visit http://creativecommons.org/licenses/by/4.0/ The Creative Commons Public Domain Dedication waiver (http://creativecommons.org/publicdomain/zero/1.0/) applies to the data made available in this article, unless otherwise stated in a credit line to the data. 
(Continued from previous page)

Results: Of 358 enrolled patients, 232 were in the development cohort (69 AKI), while 126 in the validation cohort (52 AKI). The first clinical model included the APACHE II score, serum creatinine, and vasopressor used at ICU admission. Adding sCysC and UNAG to this model improved the AUC to 0.831. Furthermore, incorporating them significantly improved risk reclassification over the predictive model alone, with cNRI (0.575) and IDI (0.085). A nomogram was then established based on the new model including sCysC and UNAG. Application of this nomogram in the validation cohort yielded fair discrimination with an AUC of 0.784 and good calibration. The DCA revealed good clinical utility of this nomogram.

Conclusions: A nomogram that incorporates functional marker (sCysC) and tubular damage marker (uNAG), together with routine clinical factors may be a useful prognostic tool for individualized prediction of AKI in septic patients.

Keywords: Acute kidney injury, Sepsis, Serum cystatin C, Nomogram, N-acetyl- $\beta$-D-glucosaminidase, Intensive care unit

\section{Key messages}

The nomogram that incorporates functional marker $(\mathrm{sCys} \mathrm{C})$ and tubular damage marker (uNAG) effectively predicts AKI risk for septic patients in ICU.

\section{Background}

Acute kidney injury (AKI) is frequent [1-3] and associated with poor prognosis [4-6]. Notably, one of the most common causes of AKI is sepsis [7] which is increasingly prevalent in critically ill patients [8-10]. Early recognition of AKI in septic patients may improve their clinical prognosis. Nevertheless, both AKI and sepsis are heterogeneous syndrome that represent multifactorial clinical conditions. In this context, early identification of AKI in septic patients remains a big challenge by using any single marker. Prior studies implied that combining markers of different characteristics may prove more accurate for AKI prediction in complex clinical settings [11-13].

Recently, combining functional and tubular damage biomarkers was reported to be an effective clinical strategy for AKI prediction, including patients suffering from heart failure [14] or those after cardiac surgery $[15,16]$. Serum cystatin $\mathrm{C}$ (sCysC) is a glomerular filtration renal biomarker, while urinary $\mathrm{N}$-acetyl- $\beta$-D-glucosaminidase (uNAG) represents tubular damage [12, 17]. NAG originates from the lysosomes of the renal proximal tubule cells and can be measured in the urine [12, 17]. Urinary NAG performed well as an early damage biomarker for AKI. In addition, previous study indicated that the increment of UNAG was caused not by sepsis, but by the occurrence of AKI [18].Moreover, both two biomarkers are clinically available [19]. Recently, we found that combining UNAG and sCysC was as an effective clinical strategy for detecting AKI in the postsurgical population [20]. Nevertheless, there is no nomogram containing this combination for predicting AKI in septic cohort.
Nomogram is used as a visualized, appreciable and intuitive tool for AKI prediction [21-23]. However, limited data exist on the nomogram for AKI prediction in septic patients. Furthermore, there are no prior nomograms incorporating uNAG or sCysC for AKI prediction in septic cohort. Therefore, this study aimed to compare the performance of AKI prediction models with or without incorporating the above-mentioned biomarkers and then develop an effective prediction nomogram for septic patients in intensive care unit (ICU).

\section{Materials and methods}

Study design and participants

This prospective observational study was conducted in the mixed medical-surgical ICU of a tertiary care hospital in China. All consecutive septic patients admitted to ICU from October 2014 to April 2019 were eligible for enrollment. The exclusion criteria included age under 18 years, presence of AKI before ICU admission, preexisting dialysis before ICU admission, end-stage renal disease (ESRD), renal transplantation, nephrectomy, refusal of consent, or missing data. The outcome was the detection of AKI within one week of ICU admission. The enrolled septic patients were divided into two cohorts in chronological order: development cohort and validation cohort. The protocol of this study was met Strengthening the Reporting of Observational Studies in Epidemiology [24] and Standards for Reporting Diagnostic Accuracy [25] criteria. 55oved by the local institutional review board.

\section{Sample and data collection}

Similar to prior studies $[19,20]$, we simultaneously collected blood and urine samples within one hour after ICU enrollment. With the standard protocol, measurements of all samples were conducted at the central laboratory of the Guangdong Provincial People's Hospital in $24 \mathrm{~h}$ after collection. The levels of uNAG and sCysC 
were measured once at the time of ICU admission. The detection of serum creatinine $(\mathrm{sCr})$ was performed at ICU admission, and thenceforth at least once a day during ICU hospitalization. We prospectively collected baseline clinical characteristics of patients and recorded the hourly urine output from ICU admission to discharge.

The following clinical variables of patient were also recorded: sex, age, body mass index (BMI), preexisting clinical conditions of each patient, admission type, baseline $\mathrm{sCr}$, baseline estimated glomerular filtration rate (eGFR),, blood laboratory values at ICU admission (hemoglobin, serum glucose, procalcitonin, lactate, Creactive protein), Acute Physiology and Chronic Health Evaluation (APACHE) II score at ICU admission, Sequential organ failure assessment score (SOFA) at ICU admission, use of nephrotoxic drugs within 5 days before ICU admission (nonsteroidal anti-inflammatory drug, angiotensin-converting enzyme inhibitor, angiotensin receptor blocker, immunosuppressant, sulfadiazine, aminoglycoside, vancomycin, acyclovir, amphotericin, allopurinol, or polymyxin), administration of radiographic contrast before ICU admission, site of infection, mean arterial pressure at ICU admission, use of vasopressor at ICU admission (dopamine, norepinephrine, or vasopressin), length of ICU stay, length of hospital stay, renal replacement therapy (RRT) during ICU stay, 30day mortality after ICU admission. The baseline estimated glomerular filtration rate (eGFR) was calculated by the Chronic Kidney Disease Epidemiology Collaboration Eq. [26].

\section{Definitions}

The outcome was the development of AKI. According to the Kidney Disease Improving Global Outcomes (KDIGO) criteria for AKI [27], AKI was defined within one week after ICU admission as any of the following: increase in $\mathrm{sCr}$ by $\geq 0.3 \mathrm{mg} / \mathrm{dl}(\geq 26.5 \mu \mathrm{mol} / \mathrm{l})$ within $48 \mathrm{~h}$, or increase in $\mathrm{sCr}$ to $\geq 1.5$ times baseline within one week, or urine output $<0.5 \mathrm{ml} / \mathrm{kg} / \mathrm{h}$ for $6 \mathrm{~h}$. Severe AKI was defined as KDIGO stage 2 or stage 3 AKI. A baseline creatinine was defined by the following rules ranked in the descending order of preference as previously recommended [28]: (1) the most recent pre-ICU creatinine value between 30 and 365 days before ICU admission ( $\mathrm{n}=58)$; (2) a stable pre-ICU creatinine value $>365$ days for patients aged $<40$ years, (the stable creatinine value was defined as within $15 \%$ of the lowest ICU measurement) before ICU admission $(n=1)$; (3) pre-ICU creatinine value $>365$ days before ICU admission and less than the initial $\mathrm{sCr}$ at ICU admission $(\mathrm{n}=$ 14); (4) a pre-ICU creatinine value (between 3 and 39 days before ICU admission) less than or equal to the initial on-admission creatinine to ICU and not distinctly in AKI ( $\mathrm{n}=153)$; (5) the lowest creatinine value upon initial admission to ICU $(n=54)$, the last ICU value $(n=51)$, or the minimum value at follow-up up to 365 days $(\mathrm{n}=27)$. Sepsis was defined according to the American College of Chest Physicians and the Society of Critical Care Medicine Consensus Conference Committee guidelines [29].

\section{Biomarker assays}

According to the manufacturer's instructions, serum Cystatin $\mathrm{C}(\mathrm{CysC})$ and creatinine, urinary creatinine, and NAG levels were measured using the UniCel DxC 800 Synchron System (Beckman Coulter, USA). The coefficients of intraassay and interassay variation for $\mathrm{sCysC}$ were $\leq 10 \%$ and $<5 \%$, respectively. The coefficients of intraassay and interassay variation of UNAG were both $\leq 10 \%$. The value of urinary NAG was normalized to urinary creatinine concentration. The personnel measuring the biomarkers were blinded to all patient's clinical characteristics. The stability of sCysC and uNAG has already been proven [30-32], thereby pre-analysis about the influence of cooling or freezing of these samples was not performed.

\section{Sample size consideration}

The sample size was calculated based on the rule described by Harrell, Vittinghoff, Steyerberg [33], namely, events per variable (EPV) being ten or greater was an important issue for estimation of multivariable regression coefficients in the multivariate regression model. Based on previous similar studies [19, 34], we considered about 5-6 significant clinical risk factors in developing a risk model of AKI. Therefore, it would need a minimum sample size of $50-60(5 * 10-6 * 10)$ patients who had events (AKI) after ICU admission in development cohort.

\section{Statistical analysis}

SPSS version 13.0 (SPSS, Chicago, IL, USA), R version 3.3.1 (R Foundation for Statistical Computing, Vienna, Austria), and MedCalc version 12.5.0 (MedCalc Software, Ostend, Belgium) software programs were used for statistical analysis.

Non-normally distributed continuous variables were presented as medians (interquartile range). The nonnormally distributed continuous variables were compared by Wilcoxon rank-sum test or Kruskal-Wallis test for one-way analysis of variance. If the Kruskal-Wallis test showed statistical significance, a post hoc test was subsequently conducted with the Bonferroni correction. Categorical variables were expressed as number (percentage) and Chi-square or Fisher's exact test was then used to compare the categorical variables. Nonparametric Spearman's test was used to assess the correlations among continuous variables displayed nonnormal distributions or categorical variables. 
In order to assess the discrimination capability of clinical models for AKI prediction, receiver-operating characteristic (ROC) curve was generated. The area under the curve (AUC) was then derived from the ROC curve. All confidence interval (CI) presented are $95 \%$. The comparison of AUC between the groups in same data set was conducted with the method developed by DeLong et al. [35], and the comparison of AUC between the groups was conducted using Hanley-McNeil methods [36]. The optimal cut-off value for predicting AKI was defined according to the Youden's index [37]. The specificity and sensitivity, negative and positive predictive values (NPV and PPV), and negative and positive likelihood ratios (LR) were also calculated.

To construct a predictive nomogram for the probability of AKI in septic patients, we firstly conducted univariate and multivariate logistic regression to construct the clinical models in the development cohort. The candidate variables with $P<0.10$ in univariate analysis were included in multivariate analysis for further variable selection. The forward stepwise (likelihood ratio) was then used. Since the APACHE II score system not only overlaps with the SOFA score but also contains more evaluating parameters than the SOFA score. To avoid the collinearity and over-fitting, the APACHE II score but not the SOFA score was included in logistic regression. A clinical model was firstly constructed without candidate variables of UNAG and $\mathrm{sCysC}$ in univariate logistic regression. Then the performance of the two biomarkers (uNAG and $\mathrm{sCysC}$ ) combined with this clinical model was compared by AUC, integrated discrimination improvement (IDI) index, and continuous net reclassification improvement (cNRI) index, as described previously $[38,39]$. A predictive nomogram was obtained from the best one. The points of each factor in the nomogram were first gotten by drawing a vertical line from the predictor to the point axis. The total points for each patient were the sum of all the points from all the factors. The estimated probability of AKI occurrence was obtained by drawing a vertical line from the total point axis to the risk of AKI prediction. The Hosmer-Lemeshow test [40] was then used to evaluate the calibration plot of the nomogram. The validity of the predictive nomogram was verified in the validation cohort. Decision curve analysis (DCA) [41, 42] was also performed to evaluate the net benefit of decision for AKI prediction with the nomogram in the entire cohort.

All the tests were two-tailed, and $P<0.05$ was considered statistically significant.

\section{Results}

Patient characteristics

Of the 713 consecutive adult septic patients screened for the inclusion in this study, 355 (49.8\%) were excluded
(Additional file 1: Fig. S1). Thus, 358 patients were enrolled for analysis, including development cohort (232 patients) and validation cohort (126 patients). Patient characteristics are present in Table 1 . In the development cohort, 69 patients (29.7\%) developed AKI after ICU admission, while 52 patients (41.3\%) developed AKI in the validation cohort. In entire cohort, there were 100 non-survivors within 30-day after ICU admission.

\section{Development of the nomogram model for AKI prediction}

The ROC curve analysis in development cohort revealed that both sCysC and uNAG predicted AKI with statistical significance (Additional file 2: Table S1). The AUCROC value of sCysC for AKI was 0.724 , which was not superior to that of uNAG. The AUC-ROC value of the combination (sCysC and uNAG) for AKI was 0.781, which demonstrated better performance than either of these two individual biomarkers.

We first analyzed the risk factors for AKI prediction without candidate variables of uNAG and $\mathrm{sCysC}$ in univariate logistic regression (Table 2). The independent risk factors included APACHE II score, serum creatinine, and vasopressor used at ICU admission. The clinical model A for AKI prediction was then constructed. This model could predict AKI with reasonable certainty (AUC-ROC $=0.784$ ). To evaluate the added contribution of these two biomarkers (uNAG and $\mathrm{s} C y s C$ ) to the clinical model for AKI prediction, logistic regression analysis was further performed (Table 3 and Additional file 3: Table S2). The AUCROC was significantly improved to 0.831 with the addition of uNAG plus sCysC $(P=0.034)$. The Hosmer-Lemeshow goodness-of-fit indicated that the risk model calibration was good $(P=0.383)$. Moreover, addition of sCysC plus uNAG significantly improved the risk reclassification of AKI over the clinical model A alone, with the largest cNRI (0.575) and IDI (0.085) (Table 3). Additionally, sCysC had significant but weak correlation with $\mathrm{sCr}$ at ICU admission $(P<$ 0.01 ), while there was no significant correlation between uNAG and $\mathrm{sCr}$ at ICU admission (Additional file 4: Table S3). Therefore, the model containing sCysC, uNAG, serum creatinine, APACHE II score, and vasopressor used at ICU admission for AKI prediction was presented as the prediction nomogram (Fig. 1). ROC-AUC analyses for AKI prediction in the development cohort were showed in the Fig. 2.

Severe AKI can also be distinguished by the prediction model which included sCysC and uNAG. The AUC for severe AKI is $0.741(0.675-0.808)$ in the entire cohort. However, there was no significant difference in nomogram score between mild and severe AKI patients (Additional file 5: Table S4). 
Table 1 Baseline characteristics and outcomes in entire cohort ${ }^{\mathrm{a}}$

\begin{tabular}{|c|c|c|c|}
\hline Characteristics & Development cohort $(n=232)$ & Validation cohort $(n=126)$ & $P$-value \\
\hline \multicolumn{4}{|l|}{ Demographic variables } \\
\hline Age, years & $62(50-72)$ & $60(51-72)$ & 0.606 \\
\hline Males, n (\%) & $143(61.6)$ & $83(65.9)$ & 0.428 \\
\hline $\mathrm{BMI}, \mathrm{kg} / \mathrm{m}^{2}$ & $22.1(20.3-23.9)$ & $22.4(19.6-25.7)$ & 0.440 \\
\hline \multicolumn{4}{|l|}{ Preexisting clinical conditions } \\
\hline Hypertension, n (\%) & $76(32.8)$ & $47(37.3)$ & 0.387 \\
\hline Diabetes mellitus, n (\%) & $34(14.7)$ & $18(14.3)$ & 0.925 \\
\hline Cerebrovascular disease, n (\%) & $63(27.2)$ & $46(36.5)$ & 0.066 \\
\hline Chronic liver disease, n (\%) & $11(4.7)$ & $2(1.6)$ & 0.220 \\
\hline Coronary artery disease, n (\%) & $23(9.9)$ & $17(13.5)$ & 0.305 \\
\hline Heart failure, n (\%) & $18(7.8)$ & $11(8.7)$ & 0.748 \\
\hline Malignancy, n (\%) & $51(22.0)$ & $29(23.0)$ & 0.823 \\
\hline CKD, n (\%) & $19(8.2)$ & $9(7.1)$ & 0.725 \\
\hline COPD, n (\%) & $28(12.1)$ & $13(10.3)$ & 0.619 \\
\hline Admission type, n (\%) & & & 0.112 \\
\hline Elective surgical, n (\%) & $38(16.4)$ & $14(11.1)$ & \\
\hline Emergency surgical, n (\%) & $44(19.0)$ & $17(13.5)$ & \\
\hline Medical, n (\%) & $150(64.7)$ & $95(75.4)$ & \\
\hline \multicolumn{4}{|l|}{ Medication before ICU admission, $\mathrm{n}(\%)$} \\
\hline Nephrotoxic drugs ${ }^{a}$ & $45(19.4)$ & $26(20.6)$ & 0.779 \\
\hline Radiographic contrast & $20(8.6)$ & 15(11.9) & 0.318 \\
\hline Sites of infection, $\mathrm{n}(\%)$ & & & 0.666 \\
\hline Pulmonary or thoracic cavity & $163(70.3)$ & $3(73.8)$ & \\
\hline Abdomen & $26(11.2)$ & $11(8.7)$ & \\
\hline Biliary tract & $5(2.2)$ & $3(2.4)$ & \\
\hline CNS infections & $19(8.2)$ & $6(4.8)$ & \\
\hline Others $^{\mathrm{b}}$ & $19(8.2)$ & $13(10.3)$ & \\
\hline MAP at ICU admission, $\mathrm{mmHg}$ & $93(84-104)$ & $90(82-101)$ & 0.096 \\
\hline Need for vasopressor at ICU admission, n (\%) & $31(13.4)$ & 25(19.8) & 0.107 \\
\hline Mechanical ventilation at ICU admission, n (\%) & 143(61.6) & $89(70.6)$ & 0.089 \\
\hline \multicolumn{4}{|l|}{ Laboratory test } \\
\hline Baseline serum creatinine, mg/dL & $0.70(0.57-0.87)$ & $0.74(0.59-0.89)$ & 0.416 \\
\hline Baseline eGFR, mL/min/1.73 m² & $96.01(81.98-111.62)$ & $97.42(80.74-110.20)$ & 0.997 \\
\hline sCysC at ICU admission, mg/L & $0.93(0.76-1.21)$ & $0.99(0.75-1.32)$ & 0.271 \\
\hline uNAG at ICU admission, U/g Cre & $44.21(24.07-74.25)$ & $34.22(20.60-61.52)$ & 0.016 \\
\hline Serum creatinine at ICU admission, $\mathrm{mg} / \mathrm{dL}$ & $0.79(0.66-1.01)$ & $0.85(0.69-1.03)$ & 0.244 \\
\hline Serum glucose at ICU admission, mg/dL & $143(112-186)$ & $150(125-211)$ & 0.060 \\
\hline Hemoglobin at ICU admission, $g / L$ & $110(93-124)$ & $106(89-124)$ & 0.420 \\
\hline Platelet at ICU admission, $10^{9} / \mathrm{L}$ & $197(139-266)$ & $188(135-264)$ & 0.903 \\
\hline Serum PCT at ICU admission,ng/ml & $0.58(0.16-2.89)$ & $0.56(0.18-2.55)$ & 0.780 \\
\hline CRP at ICU admission,mg/L & $58.66(18.33-124.48)$ & 61.30(21.90-141.18) & 0.638 \\
\hline Total bilirubin at ICU admission > 2 mg/dL, n (\%) & $30(12.9)$ & $19(15.1)$ & 0.572 \\
\hline Albumin at ICU admission $<3$ mg/dL, n (\%) & 115(49.6) & $47(37.3)$ & 0.026 \\
\hline Lactate at ICU admission > 2mmol/L, n (\%) & $64(27.6)$ & $42(33.3)$ & 0.255 \\
\hline
\end{tabular}


Table 1 Baseline characteristics and outcomes in entire cohort ${ }^{\mathrm{a}}$ (Continued)

\begin{tabular}{|c|c|c|c|}
\hline Characteristics & Development cohort $(n=232)$ & Validation cohort $(n=126)$ & $P$-value \\
\hline $\mathrm{pH}$ value at ICU admission $\leq 7.30,(\%)$ & $18(7.8)$ & $13(10.3)$ & 0.411 \\
\hline APACHE II score, at ICU admission & $19(15-24)$ & $19(15-24)$ & 0.953 \\
\hline SOFA score, at ICU admission & $5(3-7)$ & $4(3-5)$ & $<0.001$ \\
\hline$U P^{c}, \mathrm{ml} / \mathrm{kg} / \mathrm{h}$ & $1.77(1.26-2.59)$ & $1.66(1.19-2.44)$ & 0.128 \\
\hline \multicolumn{4}{|l|}{ Outcomes } \\
\hline AKI, n (\%) & $69(29.7)$ & $52(41.3)$ & 0.028 \\
\hline Severe AKI, n (\%) & $30(12.9)$ & $24(19.0)$ & 0.123 \\
\hline RRT (during ICU stay), n (\%) & $12(5.2)$ & $19(15.1)$ & 0.001 \\
\hline ICU mortality, n (\%) & $47(20.3)$ & $18(14.3)$ & 0.161 \\
\hline In-hospital mortality, n (\%) & $53(22.8)$ & $20(15.9)$ & 0.118 \\
\hline 30-day mortality, n (\%) & $64(27.6)$ & $36(28.6)$ & 0.843 \\
\hline
\end{tabular}

${ }^{a}$ The non-normally distributed continuous variables are expressed as median (25th percentile to 75 th percentile [interquartile range]). Categorical variables are

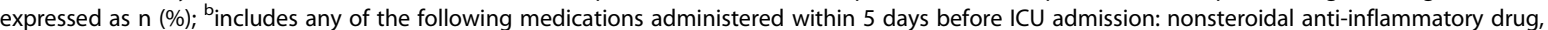
angiotensin-converting enzyme inhibitor, angiotensin receptor blocker, immunosuppressant, sulfadiazine, aminoglycoside, vancomycin, acyclovir, amphotericin,

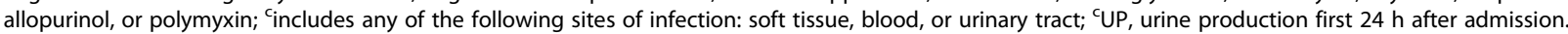
Abbreviations:AKl acute kidney injury; BMI body mass index; CKD chronic kidney disease, defined as baseline eGFR $<60 \mathrm{ml} / \mathrm{min}$ per $1.73 \mathrm{~m}^{2} ; C O P D$ chronic obstructive pulmonary disease; CNS central nervous system; MAP mean arterial pressure; ICU Intensive care unit; eGFR estimated glomerular filtration rate; sCysC serum Cystatin C; UNAG urinary N-acetyl-B-D-glucosaminidase; Cre creatinine concentration; PCT procalcitonin; CRP C-reactive protein; APACHE II Acute Physiology and Chronic Health Evaluation score; SOFA sequential organ failure assessment score; UP urine production first 24 hours after admission; RRT renal replacement therapy.

\section{Validation of the nomogram model for AKI prediction}

Based on the clinical models constructed in the development cohort, we compared the predictive ability of these two models in the validation cohort (Additional file 6: Table S5). In validation cohort, the clinical model including the APACHE II score, serum creatinine, and vasopressor used at ICU admission yielded AUC of 0.668 (95\% CI 0.570-0.765). Adding sCysC and uNAG to this model significantly improved the AUC to 0.784 (95\%CI 0,703-0.865) $(P<0.001)$. Furthermore, incorporating them significantly improved risk reclassification over the predictive model alone, with cNRI $(0.660)$ and IDI (0.104). Therefore, the model containing $\mathrm{sCysC}$, uNAG, serum creatinine, APACHE II score, and vasopressor used at ICU admission was presented as the prediction nomogram for AKI (Fig. 1). Correspondingly, ROC analysis of this model for AKI in the validation cohort was demonstrated (Fig. 2). The $P$ value for the Hosmer-Lemeshow goodness-of-fit of this model in the validation cohort were 0.541 . The AUC-ROC $(95 \% \mathrm{CI})$ for the development and validation cohort was 0.831 $(0.775-0.887)$ and $0.784(0.703-0.865)$, respectively. There was no significant difference between them $(P=$ $0.349)$. First, the clinical model was internally validated with the bootstrap validation method (Fig. 3 a). Calibration plots for the nomogram in the development (Fig. 3 a) and validation (Fig. 3b) cohort were then generated. The calibration plots demonstrated that the AKI predicted probabilities of AKI agreed with the actual probabilities.

The DCA demonstrated that nomogram could add more net benefits over the "treat-none" or "treat-all" strategies, which indicated good clinical utility of this nomogram (Fig. 4).

\section{Nomogram and adverse outcome}

Notably, there was significantly positive relationship between mortality and the total score calculated by the AKI risk nomogram (Additional file 7: Table S6). Moreover, there was significantly negative relationship between the nomogram score and the timing of AKI occurrence $(r=-0.264, P=0.003)$ (Additional file 8: Table S7).

SOFA predicted AKI in development cohort with a sensitivity of $52 \%$ and a specificity of $79 \%$, respectively (Additional file 9: Table S8). Given the correlation between SOFA score and sepsis, we further constructed a prediction model in the development cohort with SOFA score as one of the candidate variables instead of APAC HE II (Additional file 10: Table S9). A prediction model for AKI prediction was then built including serum creatinine at ICU admission, need for vasopressor at ICU admission, SOFA score, $\mathrm{sCysC}$, and uNAG (Additional file 11: Table S10). The model yielded an AUC of 0.830 in the development cohort, and 0.776 in the validation cohort (Additional file 12: Table S11). There was no significant difference between them.

\section{Discussion}

The main finding of the present study was that the prediction nomogram incorporating renal functional marker ( $\mathrm{sCysC})$ and tubular damage marker (uNAG), together with routine clinical factors may be a useful tool for 
Table 2 Logistic regression analysis of factors related to AKI in the development cohort ${ }^{\mathrm{a}}$

\begin{tabular}{|c|c|c|c|c|c|c|}
\hline \multirow[t]{2}{*}{ Variable } & \multicolumn{3}{|c|}{ Univariate analysis } & & \multicolumn{2}{|c|}{ Multivariate analysis } \\
\hline & OR unadj & $95 \% \mathrm{Cl}$ & $P$ value & $\mathrm{OR}_{\mathrm{adj}}$ & $95 \% \mathrm{Cl}$ & $P$ value \\
\hline Age, years & 1.006 & $0.989-1.025$ & 0.476 & & & \\
\hline Males & 0.622 & $0.351-1.102$ & 0.104 & & & \\
\hline $\mathrm{BMl}, \mathrm{kg} / \mathrm{m} 2$ & 0.985 & $0.901-1.077$ & 0.735 & & & \\
\hline \multicolumn{7}{|l|}{ Preexisting clinical conditions } \\
\hline Hypertension & 1.248 & $0.690-2.256$ & 0.464 & & & \\
\hline Diabetes mellitus & 1.349 & $0.626-2.907$ & 0.444 & & & \\
\hline Cerebrovascular disease & 1.696 & $0.919-3.128$ & 0.091 & & & \\
\hline Chronic liver disease & 2.044 & $0.602-6.937$ & 0.251 & & & \\
\hline Coronary artery disease & 1.956 & $0.813-4.704$ & 0.134 & & & \\
\hline Heart failure & 1.560 & $0.578-4.210$ & 0.380 & & & \\
\hline Malignancy & 0.669 & $0.326-1.374$ & 0.274 & & & \\
\hline CKD & 3.675 & $1.408-9.591$ & 0.008 & & & \\
\hline COPD & 1.137 & $0.487-2.655$ & 0.767 & & & \\
\hline Admission type, $\mathrm{n}(\%)$ & & & 0.064 & & & \\
\hline \multicolumn{7}{|l|}{ Elective surgical (reference) } \\
\hline Emergency surgical & 3.080 & $0.990-9.578$ & 0.052 & & & \\
\hline Medical & 3.300 & $1.214-8.970$ & 0.019 & & & \\
\hline \multicolumn{7}{|l|}{ Medication before ICU admission, $\mathrm{n}(\%)$} \\
\hline Nephrotoxic drugs ${ }^{b}$ & 1.084 & $0.535-2.194$ & 0.823 & & & \\
\hline Radiographic contrast & 2.073 & $0.818-5.255$ & 0.125 & & & \\
\hline Sites of infection, n (\%) & & & 0.982 & & & \\
\hline \multicolumn{7}{|l|}{ Pulmonary or thoracic cavity (reference) } \\
\hline Abdomen & 1.065 & $0.434-2.615$ & 0.891 & & & \\
\hline Biliary tract & 1.597 & $0.259-9.864$ & 0.614 & & & \\
\hline CNS infections & 0.856 & $0.292-2.508$ & 0.776 & & & \\
\hline Others $^{c}$ & 1.106 & $0.397-3.080$ & 0.847 & & & \\
\hline MAP at ICU admission, $\mathrm{mmHg}$ & 1.002 & $0.985-1.019$ & 0.833 & & & \\
\hline Need for vasopressor at ICU admission & 6.694 & $2.948-15.196$ & $<0.001$ & 5.637 & $2.349-13.528$ & $<0.001$ \\
\hline Mechanical ventilation at ICU admission & 1.925 & $0.730-5.071$ & 0.185 & & & \\
\hline Serum creatinine at ICU admission, mg/dL & 7.732 & $2.784-21.472$ & $<0.001$ & 8.955 & $2.775-28.903$ & $<0.001$ \\
\hline Serum glucose at ICU admission, mg/dL & 1.004 & $0.999-1.008$ & 0.093 & & & \\
\hline Hemoglobin at ICU admission, g/L & 0.996 & $0.985-1.007$ & 0.454 & & & \\
\hline Platelet at ICU admission, $10^{9} / \mathrm{L}$ & 0.997 & $0.994-1.000$ & 0.070 & & & \\
\hline Serum PCT at ICU admission,ng/ml & 1.014 & $0.994-1.034$ & 0.170 & & & \\
\hline CRP at ICU admission,mg/L & 1.002 & $0.998-1.006$ & 0.287 & & & \\
\hline Total bilirubin at ICU admission $>2 \mathrm{mg} / \mathrm{dL}$ & 1.994 & $0.909-4.371$ & 0.085 & & & \\
\hline Albumin at ICU admission $<3 \mathrm{mg} / \mathrm{dL}$ & 1.160 & $0.660-2.038$ & 0.606 & & & \\
\hline \multirow[t]{2}{*}{ Variable } & & \multicolumn{3}{|c|}{ Univariate analysis } & \multicolumn{2}{|c|}{ Multivariate analysis } \\
\hline & $\mathrm{OR}_{\text {unadj }}$ & $95 \% \mathrm{Cl}$ & Pvalue & $\mathrm{OR}_{\mathrm{adj}}$ & $95 \% \mathrm{Cl}$ & Pvalue \\
\hline Lactate at ICU admission $>2 \mathrm{mmol} / \mathrm{L}$ & 0.996 & $0.531-1.871$ & 0.991 & & & \\
\hline $\mathrm{pH}$ value at $\mathrm{ICU}$ admission $\leq 7.30$ & 1.198 & $0.431-3.334$ & 0.729 & & & \\
\hline APACHE \| score & 1.106 & $1.057-1.156$ & $<0.001$ & 1.104 & $1.050-1.160$ & $<0.001$ \\
\hline $\mathrm{UP}, \mathrm{ml} / \mathrm{kg} / \mathrm{h}$ & 1.142 & $0.893-1.460$ & 0.289 & & & \\
\hline
\end{tabular}


Table 3 AUC-ROC, NRI and IDI analyses of AKI in development cohort

\begin{tabular}{|c|c|c|c|c|c|c|}
\hline Variables & AUC-ROC & $P$-value ${ }^{\text {b }}$ & IDI $(95 \% \mathrm{Cl})$ & $P$-value ${ }^{\mathrm{b}}$ & cNRI $(95 \% \mathrm{Cl})$ & $P$-value ${ }^{\mathrm{b}}$ \\
\hline Clinical model $A^{\mathbf{a}}$ & $0.784(0.720-0.849)$ & & & & & \\
\hline+ UNAG & $0.817(0.759-0.876)$ & 0.091 & $0.068(0.028-0.108)$ & $<0.001$ & $0.504(0.231-0.776)$ & $<0.001$ \\
\hline$+s C y s C$ & $0.807(0.747-0.867)$ & 0.142 & $0.034(0.004-0.063)$ & 0.024 & $0.468(0.193-0.743)$ & $<0.001$ \\
\hline+ sCysC and uNAG & $0.831(0.775-0.887)$ & 0.034 & $0.085(0.042-0.128)$ & $<0.001$ & $0.575(0.303-0.847)$ & $<0.001$ \\
\hline
\end{tabular}

${ }^{\mathrm{a} C l i n i c a l}$ model A for AKI prediction is composed of serum creatinine at ICU admission, need for vasopressor at ICU admission, APACHE II score; ${ }^{\mathbf{b}}$ Versus clinical model A. Abbreviations: AKI acute kidney injury; $A U C-R O C$ area under the receiver operating characteristic curve; NRI net reclassification improvement index; $I D I$ integrated discrimination improvement index; $C I$ Confidence Interval; sCysC serum Cystatin C; uNAG urinary N-acetyl-B-D-glucosaminidase; ICU intensive care unit; APACHE II Acute Physiology and Chronic Health Evaluation score.

individualized prediction of AKI in septic patients. To the best of our knowledge, this study demonstrates for the first time that the nomogram incorporating $\mathrm{sCysC}$ and UNAG yields good discrimination for AKI prediction in septic patients.

AKI, one of the most frequent complication of critically ill patients, is especially common in septic patients [7]. In our septic cohort, AKI prevalence after ICU admission was $33.8 \%$. Hitherto, no single marker can reflect the complexity of the pathogenesis of AKI [43]. Accordingly, the ADQI working group recommended biomarkers combination for improvement in the recognition of AKI [13], including combining tubular damage and functional biomarkers. Urinary NAG represents tubular damage [12, 17], while sCysC is considered as functional biomarker [17]. Notably, they are clinically available. Moreover, $\mathrm{sCysC}$ was reported to be associated with AKI development in septic patients [44]. Additionally, previous study indicated that the increment of uNAG was caused not by sepsis, but by the occurrence of AKI [18]. Hence, we selected them to conduct our investigation based on the hypothesis that renal biomarkers can be classified as those indicating tubular damage and those representing changes in renal function [17]. In the present study, we found that compared to the clinical model without $\mathrm{sCysC}$ and $\mathrm{uNAG}$, the model incorporating them performed better for AKI prediction in septic patients. The improved ability of these two biomarkers (sCysC and uNAG) in this study is in keeping with our prior study in patients undergoing neurosurgery [20].

Therefore, we developed and validated a nomogram for AKI risk prediction including above-mentioned biomarkers. Previous studies reported several nomogram models in predicting AKI [21, 23], however, seldom focused on septic patients. Recently, an analysis identified predictive factors in septic patients admitted to the ICU in the first 24-hour and constructed a nomogram for AKI [45]. In that study, only traditional clinical parameters were considered without new renal biomarkers. To our knowledge, our nomogram is the first one incorporating functional and tubular damage biomarkers for AKI prediction in septic patients. Our nomogram effectively predicted AKI risk as indicted by the AUC-ROC value. The bootstrapped calibration curves also demonstrated that the prediction agreed well with the actual observation of AKI.

The foremost usage of this nomogram is to individually predict the probability of AKI occurrence in septic patients. The points of each risk factor in this nomogram were first determined by drawing a vertical line from the predictor to the point axis. Second, all the points from all the risk factors were sum up to generate the total points. Third, the estimated probability of AKI could be obtained by drawing a vertical line from the total point axis to the risk of AKI. For example, a patient who need for vasopressor at ICU admission (corresponds to 12 points) has serum creatinine at ICU admission of $0.6 \mathrm{mg} / \mathrm{dL}$ ( 5 points), uNAG at ICU admission of $98 \mathrm{U} / \mathrm{g} \mathrm{Cr}$ (10 points), sCySc at ICU admission of $1.2 \mathrm{mg} / \mathrm{L}$ (10 points), and APACHE II score of 26 (15 points). According to the proposed nomogram, the final point is calculated as the sum of scores for all risk factors $(12+5+10+10+15=52)$, predicting AKI risk of approximately $60 \%$. To validate the clinical utility of this nomogram, we employed DCA to assess the nomogram in the entire cohort. Based on threshold probability, this novel statistic method provided further insight into clinical consequences and calculated the net benefit gained from the nomogram. In the present study, the DCA indicated that the proposed nomogram had good clinical utility. Therefore, this nomogram could facilitate doctors an advisable decision before any administration of prevention or treatment.

CKD is associated with AKI occurrence [46]. However, CKD was not retained in the present risk model. The APACHE II score, including but not limited to patient's serum creatinine and chronic kidney function status, is a physiologically based system containing 12 physiological parameters. Therefore, APACHE II score is a common prediction tool of adverse outcome in ICU patients. In the present study, APACHE II was chosen as one of the independent predictors in the risk model. Probably owing to this reason, CKD was not chosen during the multivariate logistic regression in our study. 


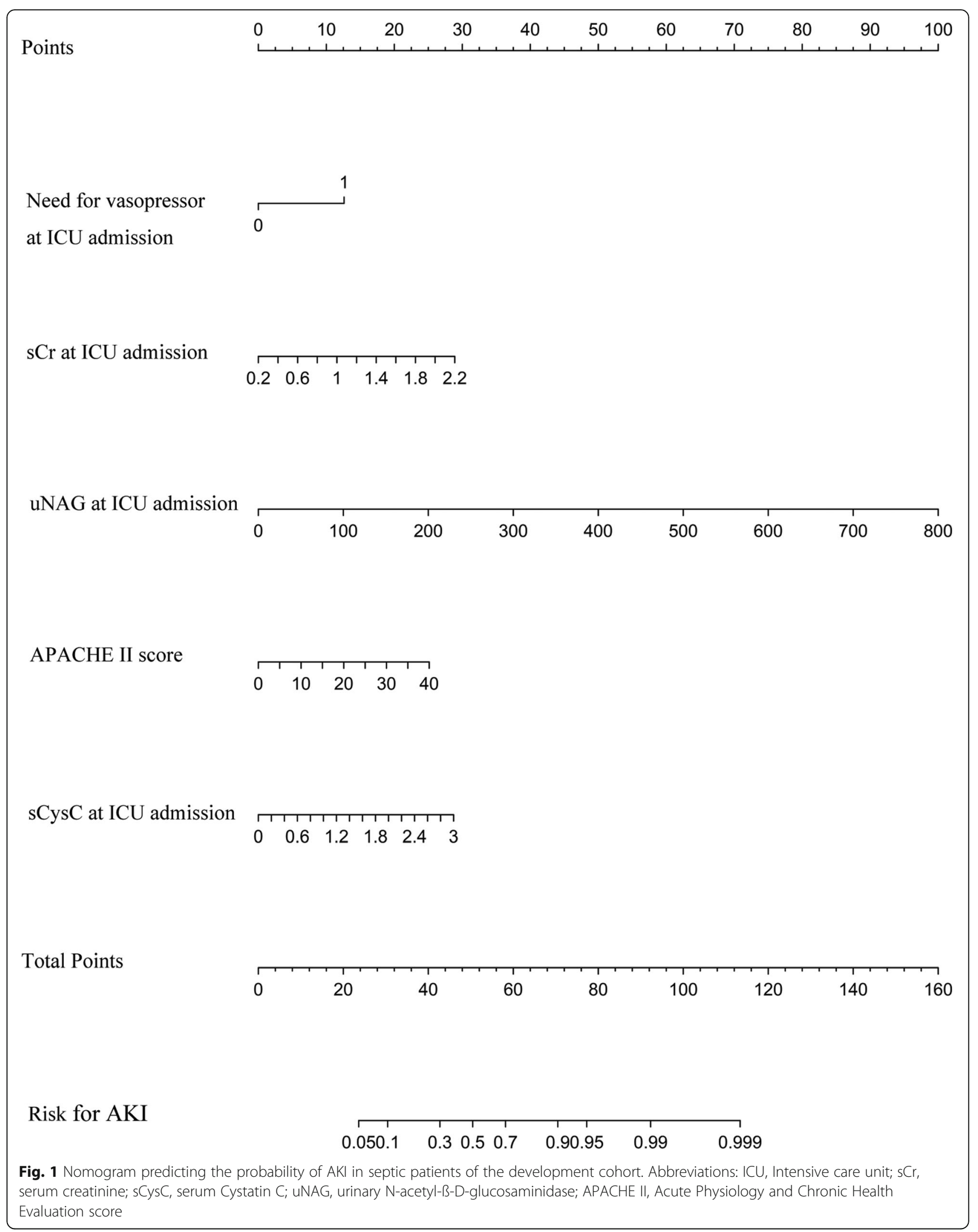




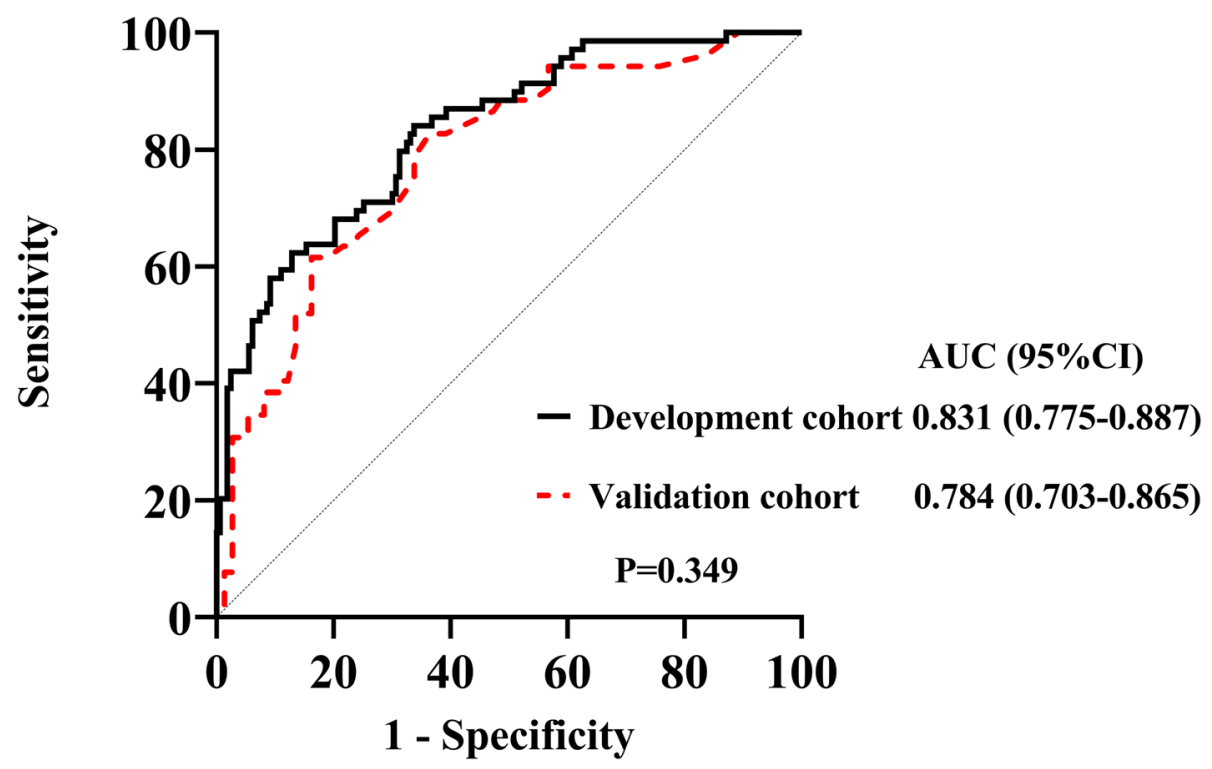

Fig. 2 Receiver operating characteristic curve analyses of model for predicting the AKI in the development and validation cohort. Abbreviations: AKI, acute kidney injury; AUC, area under the receiver operator characteristic curve; Cl, confidence interval
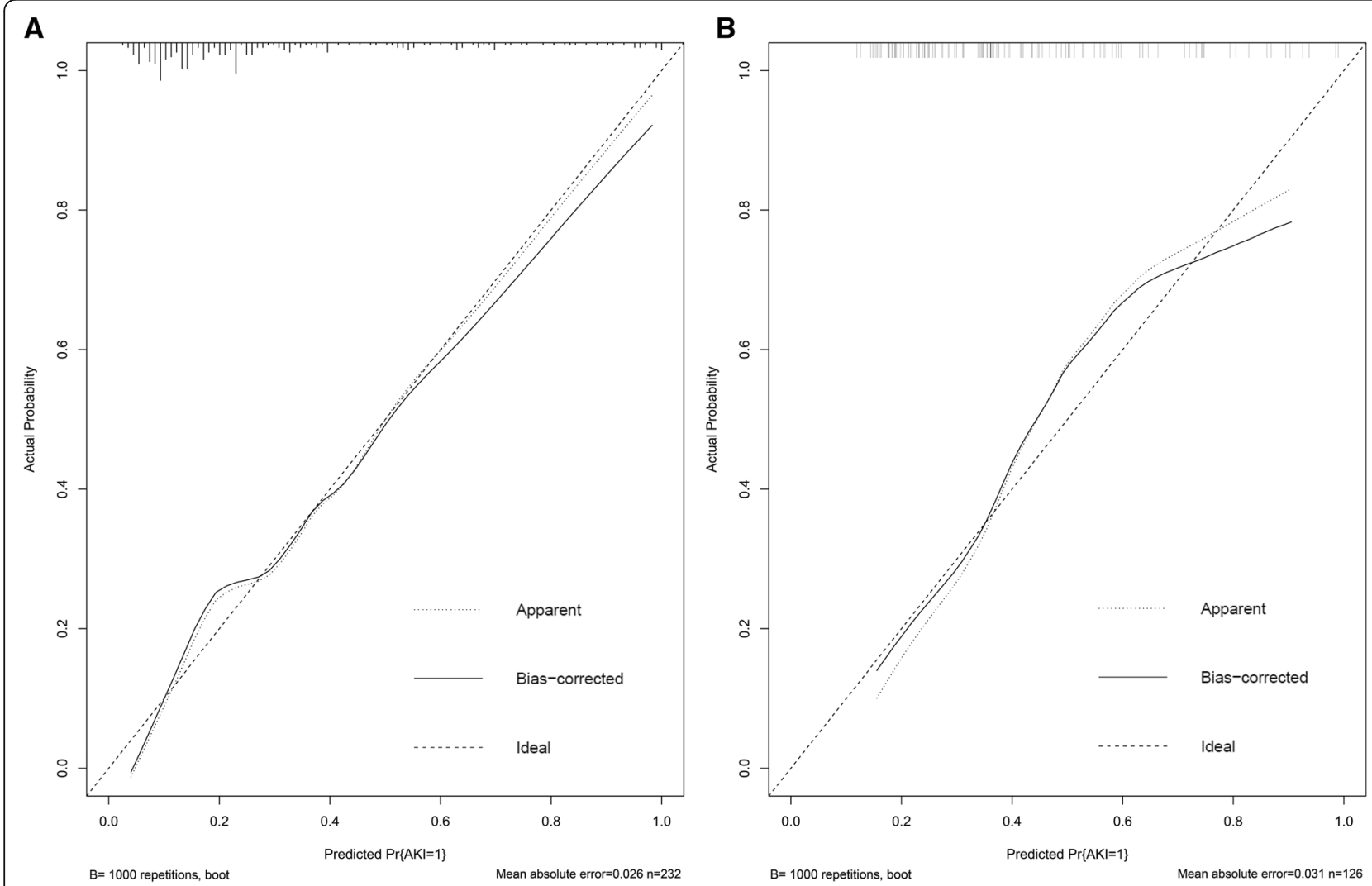

Fig. 3 Calibration plot for nomogram in the development (A) and validation cohort(B). In the calibration plot, the X-axis represents the predicted probability of AKI, and the $\mathrm{Y}$-axis indicates the actual AKI rate. The $45^{\circ}$ dashed line illustrates ideal predictions, the plot represents the accuracy of the best-fit model ("Apparent") and the bootstrap model ("Bias-corrected") for predicting AKI. The calibration plot illustrates the relationship between the predicted probability and observed probability of the scoring system for predicting AKI in the data set. Abbreviations: AKI, acute kidney injury 

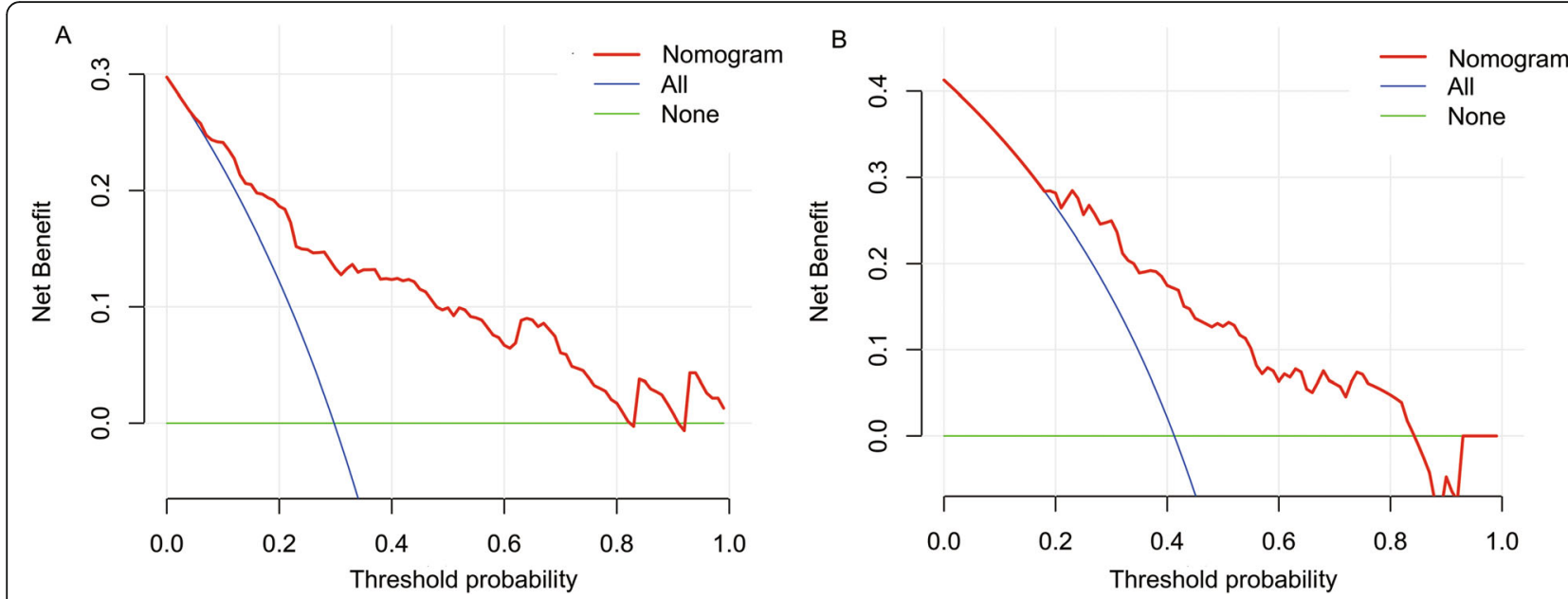

Fig. 4 DCA of the nomogram for AKI prediction in both development and validation cohorts. (A) The DCA of nomogram in development cohort; (B) The DCA of nomogram in validation cohort. The $Y$-axis shows the net benefit, and the $X$-axis indicates the threshold probability. The red line represents the nomogram. The blue line indicates the assumption that all patients are suffered from AKI and undertaken treatment. The green line represents the assumption that no patient is suffered from AKI and undertaken treatment

Our study has limitations. First, we only measured these two biomarkers once at ICU admission. According to ADQI recommendation [13], it may be not practical and cost-effective for collecting and measuring a series of samples at frequent time points. Therefore, we speculate that our conclusions are not debilitated by this limitation. Second, without an external validation dataset, we could not assess whether our nomogram may be suitable to patients outside of our center. Future study including multicenter is need. Third, there was significant but weak correlation between $\mathrm{sCysC}$ and $\mathrm{sCr}$ at ICU admission. Hence, multicollinearity of the risk factors still should be concerned in our study, even though we used multivariate logistic regression for further variable selection. Last but not least, nephrotoxins exposure was not included in our risk model. We constructed an AKI nomogram with readily available variables obtained at ICU admission for clinicians to screen the high-risk patients. Accordingly, nephrotoxins exposure after ICU admission was not taken into account in this study, which may partly contribute to the exclusion of nephrotoxins exposure. The study regarding the renal effects of nephrotoxins exposure will be conducted in future study.

The performance of AKI risk model may differ considerably across different clinical settings. Our future studies will focus on the comparison between other published models and our proposed nomogram. In addition, more recent statistical techniques, such as logistic least absolute shrinkage and selection operator (LASSO) regression need to be applied in future study.

\section{Conclusions}

The present study showed that a prediction nomogram that incorporates functional marker ( $\mathrm{sCysC}$ ) and tubular damage marker (uNAG), together with routine clinical factors may be an effective tool for individualized prediction of AKI in septic patients.

\section{Abbreviations}

AKI: Acute kidney injury; sCysC: Serum cystatin C; uNAG: Urinary N-acetyl- $\beta-$ D-glucosaminidase; sCr: Serum creatinine; ICU: Intensive care unit; ESRD: Endstage renal disease; BMI: Body mass index; eGFR: Estimated glomerular filtration rate; APACHE II: Acute Physiology and Chronic Health Evaluation score; RRT: Renal replacement therapy; MDRD: Modification of Diet in Renal Disease formula; KDIGO: Kidney Disease Improving Global Outcomes criteria; AUC-ROC: Area under the receiver operating characteristic curve; Cl: Confidence Interval; PPV: Positive predictive value; NPV: Negative predictive value; (+) LR: Positive likelihood ratio; (-) LR: Negative likelihood ratio; NRI: Net reclassification improvement index; IDI: Integrated discrimination improvement index; DCA: Decision curve analysis; DM: Diabetes mellitus; CAD: Coronary artery disease; COPD: Chronic obstructive pulmonary disease; HF: Heart failure; CKD: Chronic kidney disease, defined as baseline eGFR<60 ml/min per $1.73 \mathrm{~m}^{2}$; PCT: Procalcitonin; CRP: Creactive protein; SOFA: Sequential organ failure assessment score; UP: Urine production first 24 hours after admission

\section{Supplementary Information}

The online version contains supplementary material available at https:/doi. org/10.1186/s12882-021-02388-w.

Additional file 1: Supplementary Figure 1.Flow chart from recruitment to outcome. Abbreviations: ICU, intensive care unit; AKI, acute kidney injury.

Additional file 2: (Table S1.) Predictive characteristics of two biomarkers and their combination for AKI prediction in the development cohort. 
Additional file 3: (Table S2.) Multivariate Logistic regression analysis of factors related to AKI in the development cohort.

Additional file 4: (Table S3.) Correlations among the risk factors in the prediction model for AKI in the development cohort.

Additional file 5: Table S4. Nomogram for predicting severe AKI. Additional file 6: (Table S5.) AUC-ROC, NRI and IDI analyses of AKI in the validation cohort.

Additional file 7: (Table S6.) Correlations between AKI and mortality in entire cohort.

Additional file 8: (Table S7.) Correlation between the total score calculated from the nomogram and thedayof AKloccurrence after ICU admission.

Additional file 9 (Table S8.) Predictive characteristics of SOFA and APCHE II for AKI prediction in the development cohort.

Additional file 10: (Table S9.) Logistic regression analysis of factors related to AKI in the development cohort including SOFA.

Additional file 11: (Table S10.) AUC-ROC, NRI and IDI analyses of AKI in development cohort.

Additional file 12: (Table S11.) AUC-ROC of the AKI prediction model in the development cohort and the validation cohort.

\section{Acknowledgements}

The authors would like to thank all the doctors, nurses, technicians, and patients involved at the participating center for their dedication in the study, especially statistician Zhiqiang Nie. The Office of Medical Science of the Guangdong Provincial People's Hospital (Guangdong Academy of Medical Sciences) supervised the research, including the study design, protocol, ethical issues, data collection, and case report forms.

\section{Authors' contributions}

JCM and YJD equally contributed to the design of the research and interpretation of the data; CBC, YYD, JCM and YJD contributed to the conception/design of the research and interpretation of the data, and critically revised the manuscript. JCM, YJD, HYL, XO, SLL, YFW, FY, YYD, and CBC performed the research and collected data. JCM and YJD analyzed the data. All authors contributed to the acquisition and analysis of the data, drafted the manuscript, agree to be fully accountable for ensuring the integrity and accuracy of the work. All authors read and approved the final manuscript.

\section{Funding}

Author Chunbo Chen is currently receiving a grant (\#81671963) from the National Natural Science Foundation of China, and a grant (\#DFJH2020028) under the major program of Summit Project, Guangdong Province Highlevel Hospital Construction Project of Guangdong Provincial People's Hospital, Guangdong Academy of Medical Sciences. "Author Yunjun Deng" is currently receiving the grant (\#2016A020215129) from the Science and Technology Planning Project of Guangdong Province, China" and grant from the Guangdong Provincial People's Hospital (\#KY012020396).

\section{Availability of data and materials}

The cohorts generated and/or analyzed during this study are not publicly available, due to currently ongoing research studies, but the data are available from the corresponding author on reasonable request.

\section{Declarations}

Ethics approval and consent to participate

The Ethics Committee of the Guangdong Provincial People's Hospital approved the current study. All methods of this study were carried out in accordance with relevant guidelines and regulations. The ethics committee of the Guangdong Provincial People's Hospital supervised the study, including its design, protocol, ethical issue, and data and sample collection. Written informed consent was obtained from each patient or from appropriate surrogates for patients unable to consent.

\section{Consent for publication}

Not applicable. No individual personal data is included in the study.

\section{Competing interests}

The authors declare that they have no competing interests.

\section{Author details}

'Department of Nephrology, Guangdong Provincial People's Hospital, Guangdong Academy of Medical Sciences, 510080 Guangzhou, Guangdong, PR China. ${ }^{2}$ Department of Critical Care Medicine, Guangdong Provincial People's Hospital, Guangdong Academy of Medical Sciences, 510080 Guangzhou, Guangdong Province, PR China. ${ }^{3}$ Department of Pharmacy, Guangdong Provincial People's Hospital, Guangdong Academy of Medical Sciences, 106 Zhongshan Er Road, 510080 Guangzhou, Guangdong, PR China. ${ }^{4}$ Department of Intensive Care Unit of Cardiovascular Surgery, Guangdong Cardiovascular Institute, Guangdong Provincial People's Hospital, Guangdong Academy of Medical Sciences, 106 Zhongshan Er Road, 510080 Guangzhou, PR China. ${ }^{5}$ The Second School of Clinical Medicine, Southern Medical University, 510280 Guangzhou, Guangdong, PR China.

Received: 5 February 2021 Accepted: 26 April 2021

Published online: 13 May 2021

\section{References}

1. Li PK, Burdmann EA, Mehta RL. Acute kidney injury: global health alert. Kidney Int. 2013:83(3):372-6.

2. Xu X, Nie S, Liu Z, Chen C, Xu G, Zha Y, et al. Epidemiology and Clinical Correlates of AKI in Chinese Hospitalized Adults. Clin J Am Soc Nephrol. 2015:10(9):1510-8.

3. Spapen $H$, van Laethem J, Hites $M$, Verdoodt $A$, Diltoer $M$, Honoré PM. Treatment of Ventilator-associated Pneumonia with High-dose Colistin Under Continuous Veno-venous Hemofiltration. Journal of translational internal medicine. 2019:7(3):100-5.

4. Chertow GM, Burdick E, Honour M, Bonventre JV, Bates DW. Acute kidney injury, mortality, length of stay, and costs in hospitalized patients. J Am Soc Nephrol. 2005;16(11):3365-70.

5. Coca SG, Singanamala S, Parikh CR. Chronic kidney disease after acute kidney injury: a systematic review and meta-analysis. Kidney Int. 2012;81(5): 442-8.

6. Parikh CR, Devarajan P. New biomarkers of acute kidney injury. Crit Care Med. 2008:36(4 Suppl):S159-65.

7. Uchino S, Kellum JA, Bellomo R, Doig GS, Morimatsu H, Morgera S, et al. Acute renal failure in critically ill patients: a multinational, multicenter study. Jama. 2005:294(7):813-8.

8. Walkey AJ, Lagu T, Lindenauer PK. Trends in sepsis and infection sources in the United States. A population-based study. Annals of the American Thoracic Society. 2015;12(2):216-20.

9. Chen YJ, Chen FL, Chen JH, Wu MM, Chen YL, Chien DS, et al. Epidemiology of sepsis in Taiwan. Medicine. 2019;98(20):e15725.

10. Poston JT, Koyner JL. Sepsis associated acute kidney injury. BMJ (Clinical research ed). 2019;364:k4891.

11. Kokkoris S, Parisi M, loannidou S, Douka E, Pipili C, Kyprianou T, et al. Combination of renal biomarkers predicts acute kidney injury in critically ill adults. Ren Fail. 2012;34(9):1100-8.

12. Ostermann M, Joannidis M. Acute kidney injury 2016: diagnosis and diagnostic workup. Crit Care. 2016;20(1):299.

13. McCullough PA, Shaw AD, Haase M, Bouchard J, Waikar SS, Siew ED, et al. Diagnosis of acute kidney injury using functional and injury biomarkers: workgroup statements from the tenth Acute Dialysis Quality Initiative Consensus Conference. Contrib Nephrol. 2013;182:13-29.

14. Yang $\mathrm{CH}$, Chang $\mathrm{CH}$, Chen TH, Fan PC, Chang SW, Chen CC, et al. Combination of Urinary Biomarkers Improves Early Detection of Acute Kidney Injury in Patients With Heart Failure. Circulation journal: official journal of the Japanese Circulation Society. 2016;80(4):1017-23.

15. Basu RK, Wong HR, Krawczeski CD, Wheeler DS, Manning PB, Chawla LS et al. Combining functional and tubular damage biomarkers improves diagnostic precision for acute kidney injury after cardiac surgery. J Am Coll Cardiol. 2014;64(25):2753-62.

16. Prowle JR, Calzavacca P, Licari E, Ligabo EV, Echeverri JE, Bagshaw SM, et al. Combination of biomarkers for diagnosis of acute kidney injury after cardiopulmonary bypass. Ren Fail. 2015;37(3):408-16. 
17. Charlton JR, Portilla D, Okusa MD. A basic science view of acute kidney injury biomarkers. Nephrol Dial Transplant. 2014;29(7):1301-11.

18. Yamashita T, Doi K, Hamasaki Y, Matsubara T, Ishii T, Yahagi N, et al. Evaluation of urinary tissue inhibitor of metalloproteinase- 2 in acute kidney injury: a prospective observational study. Critical care (London, England). 2014;18(6):716.

19. Deng Y, Chi R, Chen S, Ye H, Yuan J, Wang L, et al. Evaluation of clinically available renal biomarkers in critically ill adults: a prospective multicenter observational study. Critical care (London, England). 2017;21(1):46.

20. Deng Y, Ma J, Hou Y, Zhou D, Hou T, Li J, et al. Combining Serum Cystatin C and Urinary N-Acetyl-Beta-D-Glucosaminidase Improves the Precision for Acute Kidney Injury Diagnosis after Resection of Intracranial SpaceOccupying Lesions. Kidney \& blood pressure research. 2020;45(1):142-56.

21. Hu X, Zhuang XD, Li Y, Li FF, Guo Y, Du ZM, et al. A Nomogram to Predict Contrast Induced Nephropathy in Patients Undergoing Percutaneous Coronary Intervention. International heart journal. 2017;58(2):191-6.

22. Guan C, Li C, Xu L, Zhen L, Zhang Y, Zhao L, et al. Risk factors of cardiac surgery-associated acute kidney injury: development and validation of a perioperative predictive nomogram. Journal of nephrology. 2019;32(6):93745.

23. Zhou X, Sun Z, Zhuang Y, Jiang J, Liu N, Zang X, et al. Development and Validation of Nomogram to Predict Acute Kidney Injury in Patients with Acute Myocardial Infarction Treated Invasively. Scientific reports. 2018;8(1): 9769.

24. von Elm E, Altman DG, Egger M, Pocock SJ, Gotzsche PC, Vandenbroucke JP. The Strengthening the Reporting of Observational Studies in Epidemiology (STROBE) statement: guidelines for reporting observational studies. Ann Intern Med. 2007;147(8):573-7.

25. Bossuyt PM, Reitsma JB, Bruns DE, Gatsonis CA, Glasziou PP, Irwig LM, et al. Toward complete and accurate reporting of studies of diagnostic accuracy: the STARD initiative. Academic radiology. 2003;10(6):664-9.

26. Levey AS, Stevens LA, Schmid CH, Zhang YL, Castro AF, 3rd, Feldman $\mathrm{HI}$ et al. A new equation to estimate glomerular filtration rate. Ann Intern Med. 2009;150(9):604-12.

27. Kidney Disease Improving Global Outcomes (KDIGO) Acute Kidney Injury Work Group. KDIGO clinical practice guideline for acute kidney injury. Kidney Int Suppl. 2012;2:1-138.

28. Endre ZH, Walker RJ, Pickering JW, Shaw GM, Frampton CM, Henderson SJ, et al. Early intervention with erythropoietin does not affect the outcome of acute kidney injury (the EARLYARF trial). Kidney international. 2010;77(11): 1020-30.

29. Bone RC, Balk RA, Cerra FB, Dellinger RP, Fein AM, Knaus WA, et al. Definitions for sepsis and organ failure and guidelines for the use of innovative therapies in sepsis. The ACCP/SCCM Consensus Conference Committee. American College of Chest Physicians/Society of Critical Care Medicine. Chest. 1992;101(6):1644-55.

30. Han WK, Wagener G, Zhu Y, Wang S, Lee HT. Urinary biomarkers in the early detection of acute kidney injury after cardiac surgery. Clinical journal of the American Society of Nephrology: CJASN. 2009:4(5):873-82.

31. Zhang Z, Lu B, Sheng $X$, Jin N. Cystatin C in prediction of acute kidney injury: a systemic review and meta-analysis. Am J Kidney Dis. 2011:58(3): 356-65

32. Filler G, Bökenkamp A, Hofmann W, Le Bricon T, Martínez-Brú C, Grubb A. Cystatin $C$ as a marker of GFR-history, indications, and future research. Clinical biochemistry. 2005;38(1):1-8

33. Steyerberg EW, Schemper M, Harrell FE. Logistic regression modeling and the number of events per variable: selection bias dominates. J Clin Epidemiol. 2011;64(12):1464-5; author reply 3-4.

34. Deng Y, Yuan J, Chi R, Ye H, Zhou D, Wang S, et al. The Incidence, Risk Factors and Outcomes of Postoperative Acute Kidney Injury in Neurosurgical Critically III Patients. Scientific reports. 2017;7(1):4245.

35. DeLong ER, DeLong DM, Clarke-Pearson DL. Comparing the areas under two or more correlated receiver operating characteristic curves: a nonparametric approach. Biometrics. 1988:44(3):837-45.

36. JA H, Radiology MBJ. A method of comparing the areas under receiver operating characteristic curves derived from the same cases. 1983;148(3): 839-43

37. Youden WJ. Index for rating diagnostic tests. Cancer. 1950;3(1):32-5.

38. Cook NR. Statistical evaluation of prognostic versus diagnostic models: beyond the ROC curve. Clin Chem. 2008:54(1):17-23.
39. Pencina MJ, D'Agostino RB, Sr., D'Agostino RB, Jr., Vasan RS. Evaluating the added predictive ability of a new marker: from area under the ROC curve to reclassification and beyond. Stat Med. 2008;27(2):157-72; discussion 207 12.

40. Hosmer DW, Lemesbow S. Goodness of fit tests for the multiple logistic regression model. Communications in Statistics - Theory Method 1980;9(10): 1043-69.

41. Vickers AJ, Elkin EB. Decision curve analysis: a novel method for evaluating prediction models. Med Decision Making. 2006:26(6):565-74.

42. Vickers AJ, Cronin AM, Elkin EB, Gonen M. Extensions to decision curve analysis, a novel method for evaluating diagnostic tests, prediction models and molecular markers. BMC Med Informatics Decision Making. 2008;8:53.

43. Sprenkle P, Russo P. Molecular markers for ischemia, do we have something better then creatinine and glomerular filtration rate? Archivos espanoles de urologia. 2013;66(1):99-114.

44. Leem AY, Park MS, Park BH, Jung WJ, Chung KS, Kim SY, et al. Value of Serum Cystatin C Measurement in the Diagnosis of Sepsis-Induced Kidney Injury and Prediction of Renal Function Recovery. Yonsei Med J. 2017;58(3): 604-12.

45. Deng F, Peng M, Li J, Chen Y, Zhang B, Zhao S. Nomogram to predict the risk of septic acute kidney injury in the first $24 \mathrm{~h}$ of admission: an analysis of intensive care unit data. Renal Failure. 2020:42(1):428-36.

46. Malhotra R, Kashani KB, Macedo E, Kim J, Bouchard J, Wynn S, et al. A risk prediction score for acute kidney injury in the intensive care unit. Nephrology 2017;32(5):814-22

\section{Publisher's Note}

Springer Nature remains neutral with regard to jurisdictional claims in published maps and institutional affiliations.

\section{Ready to submit your research? Choose BMC and benefit from:}

- fast, convenient online submission

- thorough peer review by experienced researchers in your field

- rapid publication on acceptance

- support for research data, including large and complex data types

- gold Open Access which fosters wider collaboration and increased citations

- maximum visibility for your research: over $100 \mathrm{M}$ website views per year

At $\mathrm{BMC}$, research is always in progress.

Learn more biomedcentral.com/submissions 